\title{
FROM DOCUMENTATION IMAGES TO RESTAURATION SUPPORT TOOLS: A PATH FOLLOWING THE NEPTUNE FOUNTAIN IN BOLOGNA DESIGN PROCESS
}

\author{
F.I. Apollonio ${ }^{\text {a }}$, M. Ballabeni ${ }^{\text {a }}$, S. Bertacchi ${ }^{\text {a }}$, F. Fallavollita ${ }^{\text {a }}$, R. Foschi ${ }^{\text {a }}$, M. Gaiani ${ }^{\text {a, }}{ }^{*}$ \\ ${ }^{a}$ Dept. of Architecture - Alma Mater Studiorum University of Bologna, Italy - (fabrizio.apollonio, massimo.ballabeni, \\ silvia.bertacchi, federico.fallavollita, riccardo.foschi2, marco.gaiani)@unibo.it
}

Commission II

KEY WORDS: Automatic photogrammetry, Visual simulation, Restoration, Photographic documentation, Orthophoto, 3D models, Color management, Physically-based rendering

\begin{abstract}
:
The sixteenth-century Fountain of Neptune is one of Bologna's most renowned landmarks. During the recent restoration activities of the monumental sculpture group, consisting in precious marbles and highly refined bronzes with water jets, a photographic campaign has been carried out exclusively for documentation purposes of the current state of preservation of the complex. Nevertheless, the highquality imagery was used for a different use, namely to create a 3D digital model accurate in shape and color by means of automated photogrammetric techniques and a robust customized pipeline. This $3 \mathrm{D}$ model was used as basic tool to support many and different activities of the restoration site. The paper describes the 3D model construction technique used and the most important applications in which it was used as support tool for restoration: (i) reliable documentation of the actual state; (ii) surface cleaning analysis; (iii) new water system and jets; (iv) new lighting design simulation; (v) support for preliminary analysis and projectual studies related to hardly accessible areas; (vi) structural analysis; (vii) base for filling gaps or missing elements through 3D printing; (viii) high-quality visualization and rendering and (ix) support for data modelling and semantic-based diagrams.
\end{abstract}

\section{INTRODUCTION}

Between 1563 and 1567 two renowned artists, the Sicilian architect and painter Tommaso Laureti and the Flemish sculptor born in Douai, Jean de Boulogne, also known as Giambologna, designed and built jointly the Fountain of Neptune, probably one of the most extraordinary examples of Renaissance fountains enhanced by its magnificent location, i.e. the confluence of the two central squares framed by imposed public buildings (Tuttle, 2001). Commissioned by the papal legate Cardinal Carlo Borromeo and supervised by Bishop Pier Donato Cesi, the fountain was intended to be the spectacular proof of an impressive urban water system (for a long time the only source of running water in the city), emblem of good governance and the munificence of the newly elected pope, Pius IV.

The fountain was conceived as a symmetric structure, to offer as a pure visual experience: the quadruple interaction of several sculptural groups (dolphins, nereids, scrolls, masks with shells, coats of arms, putti) progressively degrading upwards, leads to the figure of Neptune that crowns the whole. Water, gushing copiously from 38 nozzles, drives the composition and gives to the monument an idea of golden magnificence and vitality (Tuttle, 2015). The complex has a total amount of wetted surface of 250 square meters, reaches nearly 9 meters in height above the floor level of the paved square, being the statue of the god of the sea more than 4 meters. There exists four bronze statues or decorations of the same nature - one on each side and oriented towards the four cardinal points - that complete a sculptural composition of outstanding beauty.

To face the current deterioration due to various causes, but essentially the lack of maintenance, the Municipality of Bologna in 2015 promoted an impressive project for the restoration of the monument, in collaboration with the University of Bologna and the Istituto Superiore per la Conservazione ed il Restauro (ISCR)
(Comune di Bologna, 2017). The conservation project sets as focus point several conservative interventions concerning the whole complexity characterizing the monument. To face this complexity a multidisciplinary team (physicists, chemists, biologists, hydraulic engineers, structural engineers, geomatics experts, restorers, architects, architecture and art historians, computer and information scientists) was brought into play with the aim to accomplish diagnostic evaluations and restoration. Restoration activities are still ongoing, but the preliminary research phase has already been completed on the whole monument and the documentation experience will be presented here including some of the reached outcomes.

The base of the Neptune conservation project and of the related innovative Information System (IS) is a textured, very accurate and high-resolution 3D model featuring 610 million triangles and a geometric accuracy of the surface of $0.2 \mathrm{~mm}$. Using a multiscale acquisition strategy (Callieri at al., 2011), each single component was acquired with an Artec Eva Scanner by University of Bologna and Studio MCM and aligned on a global model produced with a terrestrial laser scanner acquisition (at a lower density than the former). All the subsequent 3D data processing work on the sampled data was done by ISTI-CNR using MeshLab. Unfortunately, this outstanding 3D model was available only at the end of the first diagnostic phase and presents a very complex structure, which makes not always easy the extraction of suitable tools for specific activities. To easily support many activities related to the actual state of analysis and design simulation, our group exploited a different approach employing 3D models and its outcomes as representation technique. This allow having commensurable and comparable data, simple to be integrated at any time. An additional 3D model - lighter but accurate in shape, color acquisition and visualization - was created to fulfil multiple requirements arisen during the

* Corresponding author 
analytical phase, ranging from real-time visualization and rendering up to analytical assessments.

In the framework of the conservation phase an overall photographic campaign was carried out by our team, a traditional output requested by ISCR to support the preliminary study and the whole diagnostics. Although the images acquisition was designed exclusively for documentation purposes, we reused this high-quality imagery for a complete 3D digital model construction of the fountain using automated photogrammetric techniques (Figure 1). This 3D model allowed to obtain the required different outputs with sufficiently accurate results, limited time and computational resources, thanks to the flexibility of the implemented automatic photogrammetry workflow that exploits solution developed by our group in recent years. The texture of the 3D model was updated during the works on various elements to record the changes of surface color after major cleaning intervention.

In this paper, we present methods and techniques of our 3D model construction (Section 2) and the different uses as tool to support different restoration activities, underlining for each uses the main features (Sections 3-7).

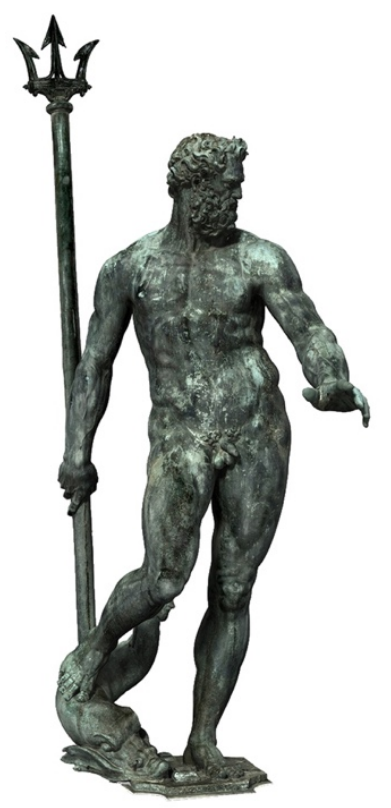

Figure 1. Render of the image-based 3D reconstruction of the statue of Neptune achieved with the customized procedure

\section{PHOTOGRAMMETRIC DATA ACQUISITION \& 3D MODEL CONSTRUCTION}

In the last years, the image-based pipeline for 3D reconstruction applications is increasingly becoming a key approach, ensuring ease of use, sufficient automation and efficient results, even for non-professional. For terrestrial applications, existing automated tools reached very high standards (Figure 2). However, although it appears that always delivering an apparently successful solution, there are still some issue: i.e. Structure-from-Motion (SfM) results, as well as for accurate color reproduction. Typical examples are Structure-from-Motion (SfM) results where, despite a message of successful image orientation and a very small re-projection error, there are some cameras wrongly oriented. A non-expert user could only spot such errors with difficultly and would proceed to the successive processing stages, negatively affecting the results.

Moreover, very few advances have been done for the problem of accurate color reproduction. Artworks as the Neptune Fountain implies outdoor environments, where natural light characteristics are extremely complex and changeable. Scenes are characterized by flat, textureless and curved surfaces reacting to light in several ways. The wide range of materials leads to different values of light reflection, porosity, etc. These difficulties increase when the problem of chromatic and tonal reproduction is addressed to the context of reality-based 3D modeling and visualization.

Furthermore, the quality of the acquired and used images is becoming fundamental to allow automated methods doing their tasks correctly. Therefore, image pre-processing methods are fundamental to improve the image quality for successful photogrammetric processing (Gaiani et al., 2016b). Finally, a typical image dataset of a $\mathrm{CH}$ object consists in a series of acquisitions observing the object through several viewing angles with some problems: not all images could contain a color chart (inaccurate colorimetric calibration); not all image areas are uniformly lighted (radiometric values can differ).

To these issues inherent to the procedure, in our case we need to add problems related to the use of a dataset not appropriate for a photogrammetric use.

The dataset reused consists of approximately 1,500 images from the complete dataset (of more than 6,000) captured for documentation. All images were taken with a Nikon 5300 SLR camera $(23.5 \times 15.6 \mathrm{~mm}$ APS-C CMOS sensor, 6,000 x 4,000 pixels with $35 \mathrm{~mm}$ nominal focal length) in a NEF file format (i.e., a 14-bit compressed RAW). To capture zenith images, an aerial work platform was used before the installation of the permanent scaffold. Pictures were taken considering the global complexity of the monument, i.e. the fountain is a sculpture in the round, characterized by numerous cavities and protrusions, and considering the lighting conditions of outdoor environments, to overcome the typical problems rooted in this type of cultural assets. The main problems of this dataset consist in:

- limited field of view of each image (the usual feature of the imagery used as support tool to plan the surfaces restoration); - limited overlap often existing between adjacent photos (this is probably the most important requirement not met);

- presence of wide-angle shots in the dataset;

- each part of the scene not always appear in at least three separate views taken from different locations;

- camera network is not optimized and sometime appears inappropriate.

Our photogrammetric pipeline has aimed to give solution to the above listed problems. The workflow starts from RAW photographs and consists of a customized, optimized and automated procedure, able to improve the traditional solution based on Structure-from-Motion, Bundle Adjustment and dense stereo matching: the first step deals with a completely automated image pre-processing, aimed at enhancing photograph quality (radiometric calibrated images) and yielding better results during the following phase of photogrammetric processing (image orientation and dense image matching) (Gaiani et al., 2016b); the second step concerns feature extraction and image matching by means of a customized SIFT detector/descriptor and a customized solution for bundle adjustment. Dense stereo matching, mesh reconstruction and texture mapping are based on the state-of-the-art commercial software Agisoft PhotoScan Pro (Agisoft, 2017) with some customizations and enhancements. Attention has been paid to reproduce the color and reflectance properties of the materials (white Istrian stone, red Verona marble and bronze) used for the statue of Neptune correctly. The enhanced workflow allows increasing the accuracy of description and the reproduction of colors starting from the extraction of the albedo map reconstruction. It consists in firstly exploiting pose and camera calibration of a suitably selected set of images and then projecting images on the mesh, producing a texture atlas. 
Both these functions are well implemented in PhotoScan, reliably starting from Lempitsky \& Ivanov (2007), allowing excellent color blending of images dataset and a compact atlas as output. In detail, we describe our key improvement.

A. SfM geometry reconstruction. Nowadays the most popular and used operator to run the initial phase of the SfM pipeline (images matching) is the SIFT method (Lowe, 2004) for interest point detection/description and the use of the Euclidean distance criteria for the matching. SIFT failures case are changes in the illumination conditions, reflecting surfaces, object/scene with strong $3 \mathrm{D}$ aspect, highly repeated structures in the scene and very different viewing angle between the images (Apollonio et al., 2014). We run an optimized implementation of the ASIFT algorithm (Morel \& Yu, 2009) aiming to correct the SIFT failures in case of very different viewing angles. For efficiency, we computed matches using the approximate nearest neighbor $\mathrm{kd}$ tree algorithm (FLANN) (Muja \& Lowe, 2009). Details on this implementation can be found in (Gaiani, 2015). Bundle Adjustment exploits a customized version of Multicore Bundle Adjustment (Wu et al., 2011) appropriate for our case study.

B. Color management and calibration. The aim of our color preprocessing essentially consists in obtaining radiometric calibrated albedo maps able to ensure the consistency and fidelity of surfaces colors reproduction at runtime in the visualization application OpenGL-based. For digital images, color characterization methods refer to the techniques of converting camera responses (e.g. RGB) to a device-independent colorimetric representation (e.g. CIEXYZ) (Green \& MacDonald, 2002). Between the two general approaches to color characterization - spectral sensitivities-based and color targetsbased (Hong et al., 2001) - the latter technique is adopted, exploiting spectrometric measurements of a target X-Rite ColorChecker Classic (CC) (McCamy et al., 1976).

The color correction settings are applied automatically using a developed software solution consisting in a RAW image processing implemented in MATLAB and supported by DCRaw, an open-source command-line program, easily coupled with MATLAB (DCRaw, 2017), allowing image demosaicing, white balance, output file in a rendered color space, gamma correction, brightness control, 8-bit/16-bit conversion. Our workflow, partially described in (Gaiani et al., 2016a), is as follows:

1. RAW image 16-bit linearization and devignetting;

2. ColorChecker localization;

3. Polynomial fitting of $\mathrm{CC}$ observed with expected values;

4. Image correction using the fitting function found at point 3;

5. White balance of the correct image;

6. $\Delta \mathrm{E}^{*}{ }_{00}$ mean error evaluation on the $\mathrm{CC}$;

7. Iterative image correction using the fitting function found at point 3 increasing the degree of the polynomial at each step (iteration stops when $\Delta \mathrm{E}^{*}{ }_{00}$ stops decreasing);

8. Image correction using the new fitting function;

9. ACR scripts calibration;

10. Color space assignment

Only at the end of the workflow, the images are compressed in the visualization color space sRGB to minimize typical problems emerging using non-linear workflows.

Color characterization is performed in two different phases:

- Captured images development and pre-processing;

- Albedo map pre-processing for rendering.

The second phase is needed to overcome a limitation of PhotoScan software where color is completely unmanaged producing unwanted artefact and - generally - color differences even considerable due to mismatches between the color spaces. Four different dataset were processed corresponding to the main levels the fountain is composed of (pool, basins, pinnacle, Neptune's statue), for better management of data processing, texture mapping, different level of detail depending on the material (metallic or stone decorations) and final rendering. The quality of the final 3D model is nearly 13 million triangles over a dense cloud of 70 million points. Albedo maps resolution is about $1 \mathrm{~mm}$ per pixel and atlases of $8,192 \times 8,192$ pixels. The model was scaled by means of reference points, measured with a Geotop GPT-2005 Total Station and georeferenced against the C60 municipal reference datum point. A summary of the final data of this model is in Table 1.

\begin{tabular}{|c|c|c|c|}
\hline & $\begin{array}{c}\text { Numb. 3D } \\
\text { points dense }\end{array}$ & $\begin{array}{c}\text { Numb. } \\
\text { triangles }\end{array}$ & $\begin{array}{c}\text { Texture size } \\
\text { (pixels) }\end{array}$ \\
\hline L5_Nettuno & $5,098,966$ & $1,013,028$ & $8,192 \times 8,192$ \\
\hline $\begin{array}{c}\text { L3_Castellum + } \\
\text { L4_Castellum }\end{array}$ & $14,100,907$ & $2,418,613$ & $8,192 \times 8,192$ \\
\hline L2_Castellum & $19,607,988$ & $3,343,371$ & $8,192 \times 8,192$ \\
\hline $\begin{array}{c}\text { L0_Scalinata + } \\
\text { L1_Vasca }\end{array}$ & $31,286,191$ & $5,988,829$ & $8,192 \times 8,192$ \\
\hline
\end{tabular}

Table 1. Summary of final data of Neptune's fountain 3D model
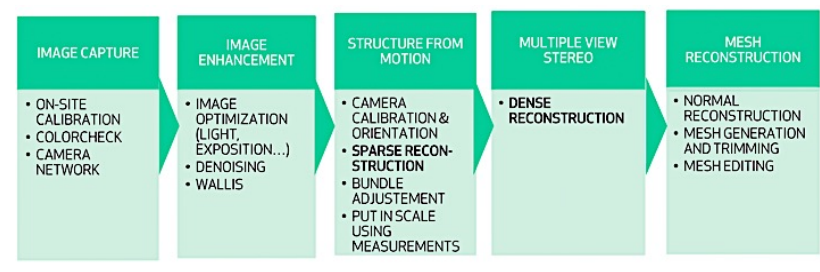

Figure 2. The pipeline aimed at the geometry reconstruction from images

\section{ORTHO-IMAGES AND CROSS-SECTIONS}

The first use of our 3D model concerned the generation of orthoimages of the main elevation views (Figure 3) and plans or cross-sections of the whole complex including the underground area (Figure 4).

Orthophotos are a very useful product for $\mathrm{CH}$ documentation since in this metric product it is possible to combine radiometric information with real measure, allowing a complete representation from every point of view (both terrestrial and aerial) of the analyzed object (Mavromati et al., 2002). In our case these representations supported instant annotations, graphical analysis, quick generation of different design solutions in different areas (structural, hydraulic, lighting).

In the process of generation of the orthoimages it is important to determine two basic issues: the accuracy and resolution of data. The resolution should be considered as the possibility to discriminate details (Markiewicz et al., 2015). It is defined by means of the pixel size of the resulting orthoimage. Our orthoimages, generated automatically using PhotoScan, have a resolution of $0.5 \mathrm{~mm}$ per pixel.

Cross-sections were a key tool to understand internal/external correspondences related to structural, hydraulic and electrical systems and for underground areas analysis and redesign. The virtual access to the underground had the purpose of providing technicians with spatial information essential for the rehabilitation of the water and electricity supply systems. In detail, these technical drawings comply with the needs of investigation and diagnostics on monumental complex: correct definition of masonry thickness, placement of metal chains and brackets that secure the Neptune to the structure for the purposes of structural inspections, positioning of the plant systems (plumbing, electrical installation, etc.). 


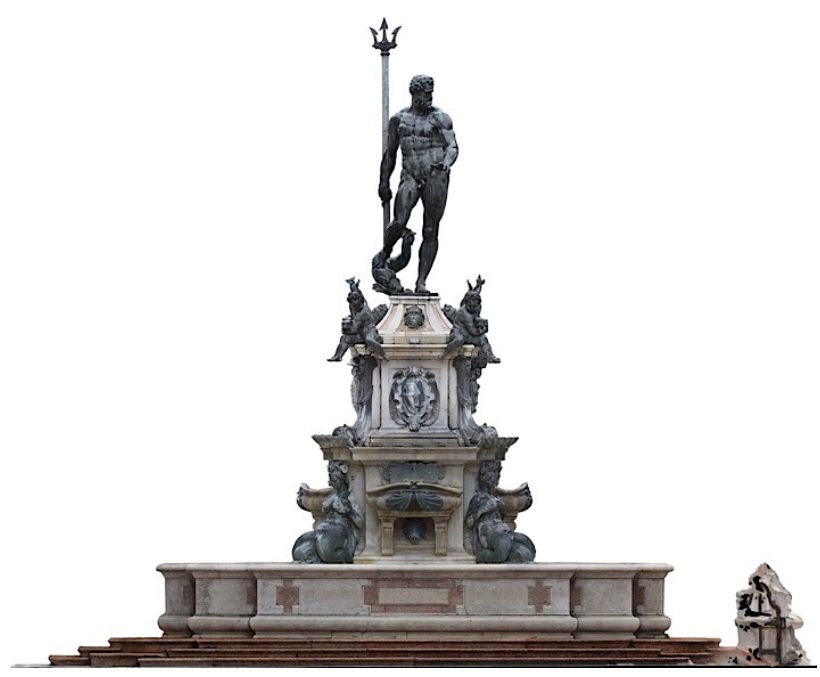

Figure 3. Orthoimage of the southern elevation of the fountain

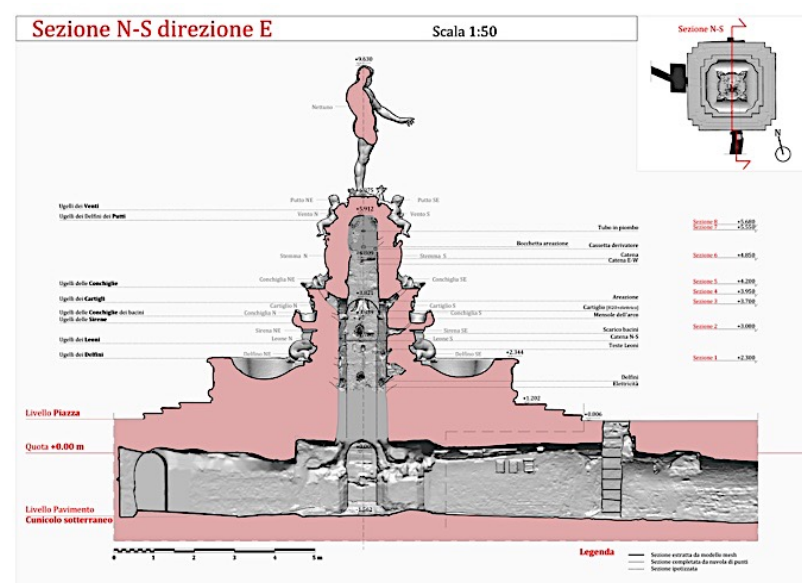

Figure 4. North-South cross-section of the whole monument, including internal and underground areas

To fill these gaps, a 3D photogrammetric reconstruction of the underground complex was completed, including the internal areas of the fountain and the narrow tunnels used, today as in the past, as technological core of the hydraulic system which supplies the fountain with treated fresh water (Figure 5). A textured version of this 3D model was also used to support decay analysis of the underground area of the fountain with the aim of an integrate analysis and redesign. The 3D model was built from a dataset of 800 high-detail images, in two different campaigns, using a Nikon D3100 (image size 4,608 x 3,072 px) equipped with an AF-S NIKKOR DX 18-55 mm f/3.5-5.6 G VR lens and a Nikon D40 SLR (image size 3,008 x 2,000 px) equipped with an AF-S NIKKOR DX 18-55 mm f/3.5-5.6 G ED II lens. This $3 \mathrm{D}$ model has been oriented with the external complex by means of active sensor technique, obtaining a general polygonal mesh of the distribution corridors and a more detailed model of the upper part inside the structure of the fountain. Basically, we used six scans from a Leica C-10 laser areas with gaps and holes existing in the laser scanning data set, due to the presence of numerous obstacles of plant systems (plumbing, electrical and metal brackets) in the underground tunnels.

Cross-section positions were selected as the most adequate crossing plane according to the aims of the analysis/inspections.

\section{SURFACE CLEANING}

Restoration activities usually involve surface cleaning as the method for removing accumulated dirt, patinas or other substances from the monument's surface and alteration of materials by environmental or chemical pollution. Checking the irreversible process of cleaning is a delicate task, that requires a work of synthesis between theoretical knowledge and practical experience to define an effective operating protocol on a limited patch area to be extended later to the entire artefact's surface.

Today, solutions based on chemical evaluation of the type of material removed are not quickly available to the restorer during the cleaning work and consist in micro-destructive techniques. Other solution based on visual examination aided with digital microscopes presents also several problems: it is not easy to find the same point in different cleaning phases, the inspection concerns a very limited area, color reproduction is not accurate. Finally, to understand what is the proper layer we need not only the experience gained by the restorer and specific tests, but also to cross heterogeneous data coming from different sources and fields of knowledge (restoration, chemistry, art history, etc.).

To overcome the complex, long and tedious, sometime inaccurate procedure and to have an accurate check of the progress of cleaning work, we developed a new, quick $3 \mathrm{D}$ photogrammetrybased solution allowing to compare interactively side-by-side in an OpenGL viewer the bronze surfaces before, during and after the cleaning treatment (Figure 6) (Apollonio et al., 2017).

The solution uses documentation images, and exploits only the restorer expertise and visual ability.

The new software allows the conservators to assess in real time and with a high level of fidelity in color and shape, the 'surfaces' to be cleaned before, during and after the clear-out treatment. The solution besides allows an effective and valuable support tool for restorers to identify the original layer of the bronze surface.

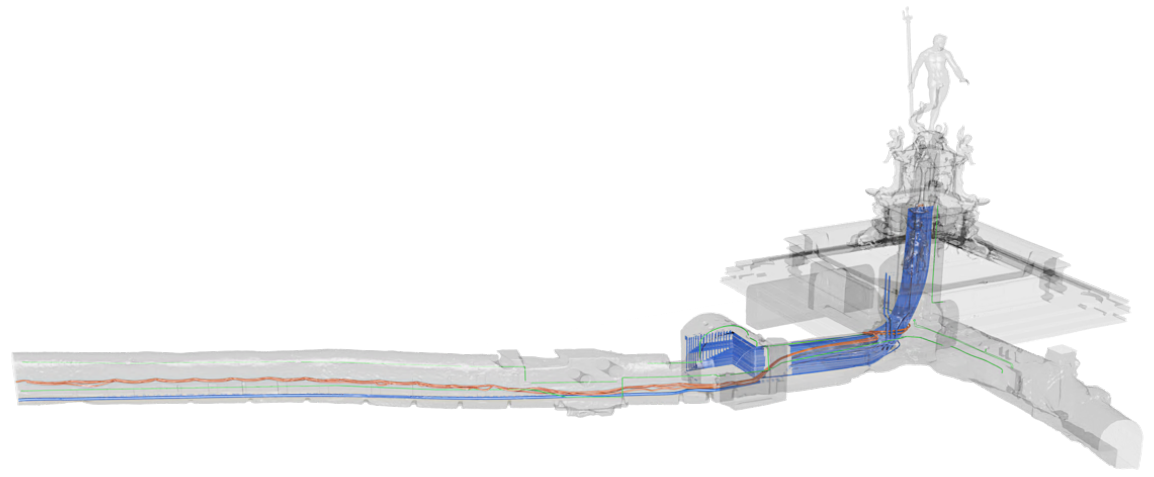

Figure 5. 3D model of the fountain and the subterranean area, with the plant systems clearly visible: water supply (blue), electricity grid (green and orange) 


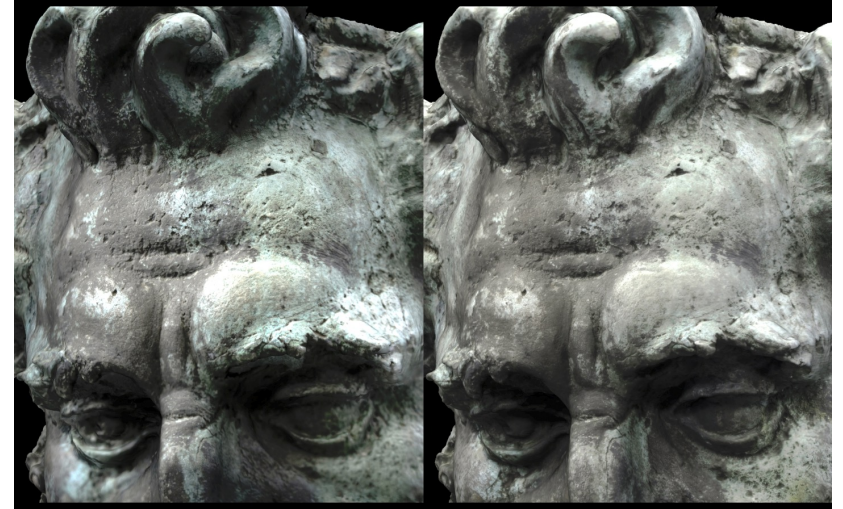

Figure 6. Side-by-side real-time visualization of the Neptune head, in two different cleaning phases

\section{WATER SYSTEM SIMULATION}

A key problem in the ongoing restoration yard of the Neptune Fountain is the rehabilitation of the water supply system and water jets. The use of the 3D model was twofold:

- To support the study of the hydraulic system of the fountain, originally supplied of spring water by means of an aqueduct specially designed for the purpose and today, after changes started in the late nineteenth century, connected to the new urban aqueduct. It consists in a complex underground structure of tunnels and pipes, severely damaged by time and by human negligence, and is the heart of the entire system;

- The rehabilitation of the design of the jet system based not only on texts and written information by Laureti, their inventor, but also on a beautiful drawing by Marcantonio Chiarini, dating back to 1763 and illustrating a possible configuration and, finally, on some mathematical studies carried out in 2003 regarding the effective flow of the system. For several reasons, it is extremely difficult to study and simulate the activation of the jets of water in a historical fountain, test their trajectories and formulate a design proposal. These difficulties include: damage to parts of the system, temporal superimposition with other studies and interventions, the need to avoid accidental deterioration of the original materials, and the fact that different solutions had to be tested and verified.

A team of five specialists (fluid dynamics engineer, hydraulics engineer, environmental engineer, material engineer and biologist) developed a new design of the hydraulic system and an appropriate jet system capable of restoring the fountain's original image. Authors developed a whole model of the pipes and a computer graphics simulation to assist the assessment of jets designed achieved using a physical-mathematical formulation of the layout of the jets of water and how they interact with the existing monumental complex. According to the information derived from historical sources and drawings, along with the calculation of the water flow rate and the trajectories of spurts, the model simulates the water flow, presenting an accurate and plausible reconstruction (Figure 7).

Simulation of the water system of the Neptune Fountain, in practice, required to model three phenomena concerning the behavior of fluids as motion systems using computer graphics techniques:

a. laminar jets of water coming out of the nozzles;

b. water in the main and secondary basins;

c. interaction of the jets with the basin.

The 3D model simulation allowed us to produce a realistic model of the jets of water in just a few days and without touching the monument (Apollonio et al., 2016). The result was a full HD format animation $(1,920 \times 1,080$ pixels) made up of 2,200 still frames for a duration of 1 minute and 30 seconds (Figure 8). It is made up of three sequences created using the classic key-framing technique. The sequences were used to illustrate:

- the different impact of the monument with and without the jets of water;

the overall functioning of the system;

- the functioning of specific areas as perceived during real observation and according to the preferential picture planes described so well in Richard Tuttle's book (Tuttle, 2015).

The animation was recalculated and modified four times to reflect the changes made by the various specialists involved in the project. Mainly, the water jets simulation allowed an iterative optimization of the project. Observing the animation, hydraulic and fluid dynamics engineers verified the current directions of the jets and progressively corrected the illogical ones.

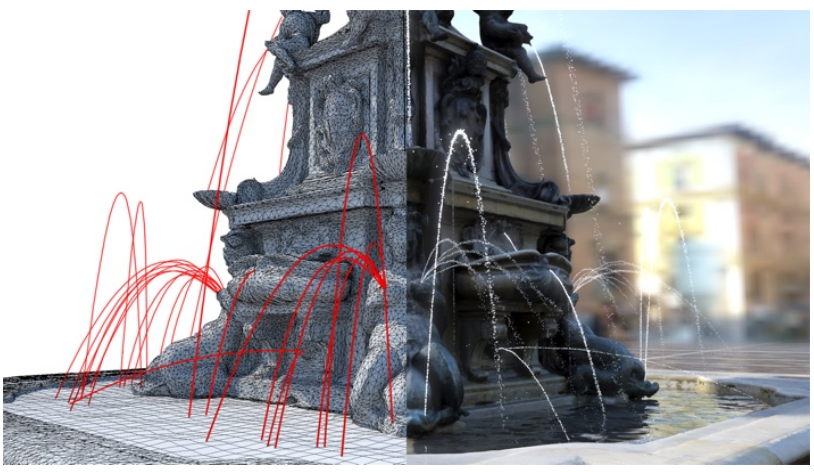

Figure 7. Detail of the simulated trajectories of water spurts into the marble pool

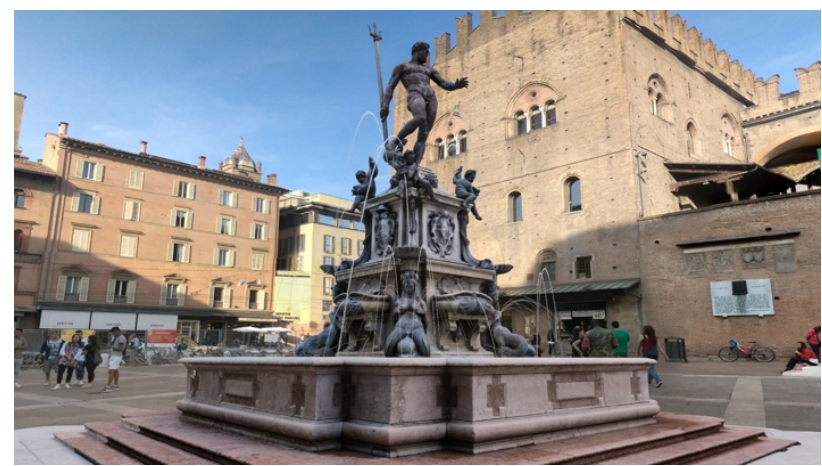

Figure 8. A frame of the virtual simulation of the Neptune Fountain having all water jets reactivated

\section{LIGHTING SIMULATION}

In the whole conservation project for the Neptune Fountain the only novelty, with respect to the concept designed by Giambologna and Laureti and how we know the monument today, concerns the artificial illumination project. New lighting will provide its new image at night, at events and on winter days. The best way to provide a lighting project is to simulate it using digital media (Rossi et al. 2004). For a realistic depiction of architectural scenes, it is important to perform a physically based simulation of light transport and to use a physically-based rendering system tailored to the demands of lighting design and architecture (Pharr \& Humphreys 2016).

The lighting simulation was carried out in collaboration with Laboratorio LUCE (Department of Design, Politecnico di Milano), thanks to their relevant scientific and technical expertise in the field, using our 3D textured model as base for the new lighting simulation (Figure 9). 


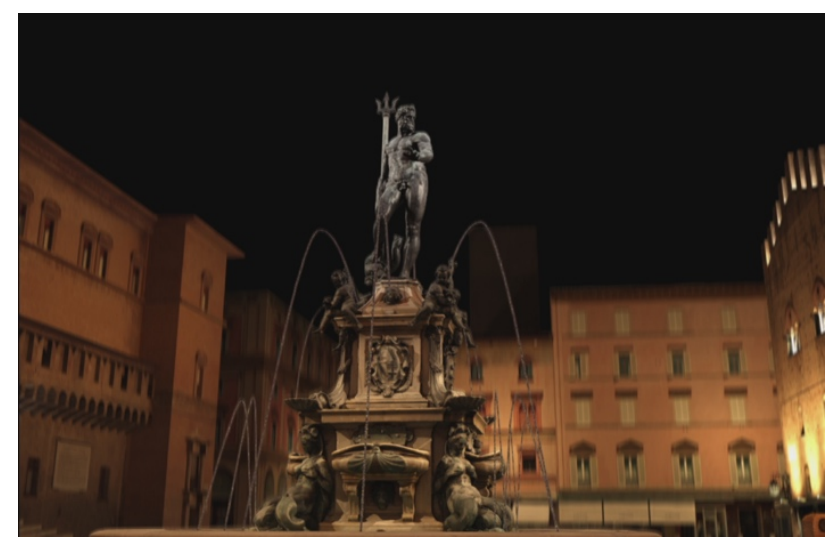

Figure 9. Frame of the virtual simulation for the study and verification of new lighting for night-time or special events

The three concepts were communicated and checked exploiting many rendered images and three computer animations, simulating three different solutions able to fulfil the requirements of the Municipality and suitable levels of luminance. The first and second lighting concept have a more static design, only changing the illumination of the statue at predetermined time intervals, while the third provides a more flexible solution for a dynamic managing of the monument lighting.

The 3D model, in fact, has been used for virtually verifying different illuminations solutions and checking the many lighting impact: unwanted shadows casted on surrounding buildings; possible illumination effect depending on the reflectance of the marble and bronze surfaces after restoration; the effect of different color spotlights (blue, pink, red, violet) to be used in special events; the effect of the illumination of the water jets; the changes in the global design of the square adding different type of lighting on the pavement.

\section{STRUCTURAL ANALYSIS SUPPORT}

Multiscale structural analysis of the fountain was an important issue encountered. The built 3D model allowed in this case to support multiple activities, otherwise very hard to be faced:

Global seismic analysis;

- Structural analysis concerning specific parts of the complex: e.g. the control room in the underground;

Analysis concerning specific geometric features: volumes, areas, centers of gravity;

Analysis concerning the construction features directly visible (e.g. anchorages of marble slabs to the brick structure).

In this sense, contribution was mainly addressed to the process of knowledge, that has as its objective the acquisition of information necessary to the characterization of geometric properties of the

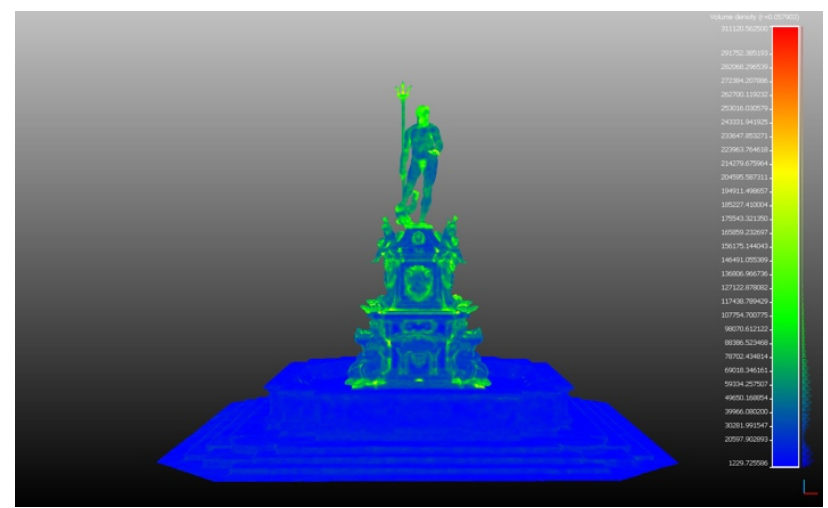

Figure 10 . Volume density computing on the 3D model structure to model the relationships among the elements that constitute it, with the aim of the structural interpretation (Tucci \& Guardini, 2014), rather than a mean to perform structural calculation e.g. using FEM analysis (Gonizzi Barsanti \& Guidi, 2017; Hamed \& Abdelhafiz, 2016).

In detail, in addition to the virtual exploration of higher internal areas difficult to reach and geometric measurements, specific parts extracted from the whole 3D model and remodeled at higher resolution provided a quick and reliable assessment of the thickness of the supporting structure (Figure 10).

Geometric analysis was supported by the Open Source software CNR-ISTI Meshlab (Meshlab, 2017) and CloudCompare (CloudCompare, 2017).

\section{MODELLING AND 3D PRINTING FOR RESTORATION OF GAPS AND MISSING PARTS}

A typical problem of artworks restoration is the need of integration of missing part or areas to improve the conservation of the monument. The statue of Neptune has on its surface several gaps of various sizes, usually no larger than few millimeters. A major problem appears in the case of the trident held by the divinity in his right hand: one of its extremities is missing. This is due to defects in the final stage of the melting process: for that time, in fact, an experimental technique was adopted which caused lacks on the final surface of the statues (Figure 11-bottom right).

Following the advice of the supervisor in charge of the restoration (ISCR), we decided to study an integration of the missing part of the trident using digital 3D printing techniques, able to physically model the missing parts and produce them in a fast and accurate manner, enabling restorers to manufacture perfectly matching elements for integration purposes (Scopigno, 2017). Our highpoly $3 \mathrm{D}$ model was used to support the modelling of the missing parts in a three-step process: (i) survey through digital photogrammetry; (ii) reconstruction of the missing part through surface modelling; (iii) 3D printing.

To achieve phase (ii) a hybrid modelling-based approach was adopted with the aim of combining both the accuracy of mathematical modelling and the flexibility of polygonal modelling of sculptural shapes. To do this, we used T-splines technology (Sederberg et al., 2003). A T-spline surface can be considered as a NURBS surface with the difference that you can have partial isoparms. While reference points of a NURBSsurface must form topological similarity of a rectangular frame, T-splines can have the so-called inner T-points (a reference point with three rather than four neighbors). Modeling surfaces with Tsplines can reduce the number of control points in comparison to NURBS surfaces and make pieces easier to merge. T-splines are an efficient bridge technology between NURBS and meshes.

This technique (used trough a plug-in for Rhinoceros developed by Autodesk), allowed to properly check the accuracy of the general shape and of the surface area to be glued on the existing bronze artwork (Figure 11 top). The final NURBS model was then re-converted into a STL mesh for the 3D printing.

The physical model was printed using a Stratasys Dimension Elite 3D printer and Fused Deposition Modelling (FDM) technology. The printer uses Soluble Support Technology which dissolves the supports in a water based solution, minimizing the typical problem of FDM, i.e. difficulty to print strong overhangs. The material used was ABS-M $30^{\mathrm{TM}}$, that is from $25 \%$ up to $70 \%$ stronger and with greater tensile than standard ABS. Moreover, it presents more impact and flexural strength than standard ABS; besides, layer bonding is significantly stronger than that of standard ABS, for a more durable part. To avoid the problem of visibility of layered structure generated by the deposition scheme, the printed object was finally sanded and refined. 


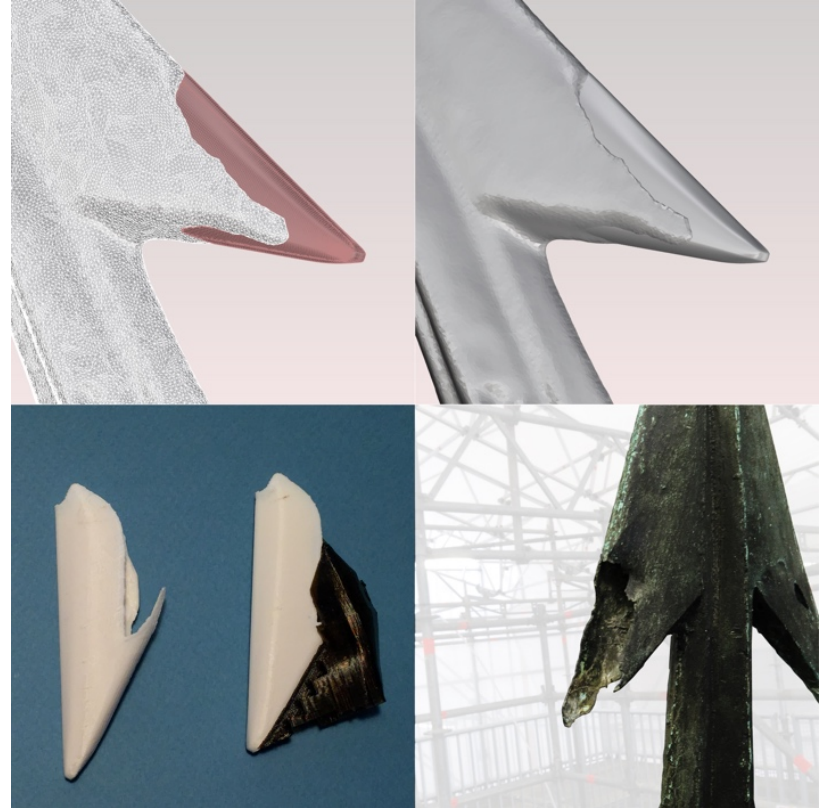

Figure 11. The final mesh model of the trident tip with the remodeled part (top); the printed part w/o and with the support (bottom left); the trident tip with the missing part (bottom right)

\section{DATA MODELLING AND SEMANTIC-BASED DIAGRAMS}

The ongoing restoration of the fountain is grounded on 3D-based Information System capable of providing innovative, efficient and user-friendly management of the entire process of collection, preservation and retrieving of information and data related to the diagnosis and the restoration. This IS is the result of a joint project between the University of Bologna, the ISTI-CNR and ISCR. The Neptune IS structure is based on a 3D model of the monument built and organized as a semantic cognitive system. This semantic organization was developed not using the final 3D model that today drive the IS, but our lighter model where subdivision in sub-elements, organized in hierarchical levels of aggregation and classifications, was modified several times to progressively include all the problems emerging from the shape and construction of the monument.

Furthermore, in anticipation of a possible/future use of the digital model within a web platform for managing the maintenance of the monument, a data modelling was organized taking into consideration different aspects, also in this case exploiting the photogrammetric 3D model. A conceptual model of the system has been done for providing: on one hand with the modelling of the structure of the IS (relationship between data, management of the operating steps and of its chronological development); on the other with the semantic structuring of the $3 \mathrm{D}$ model such as to ensure an efficient data annotation procedure.

The structure of conceptual model, based on the multidisciplinary analysis for information purposes, includes:

- different stages of cleaning procedures carried out on the monument and finally successive ordinary/emergency maintenance (Activities);

- the numerous areas of interest of the mentioned activities (Fields);

- users involved in the documentation/restoration activities (Operators);

- the possible relationships connecting existing data and the 3D model (Operations).
To achieve semantic structures, three steps mutually connected (modelling, segmentation and visualization) were carried out, with the aim of:

- Producing a textured, high-resolution reality-based 3D model;

- Organizing it according to a defined semantic structure;

- Providing visualization features and modalities that follow the needs of the operator/user.

First of all, the 3D digital model has undergone a suitable semantic partition dividing the object in different sub-elements according to a hierarchical relation among items. In fact, the conceptual architecture of the IS needs to hierarchically organize data and 3D objects within the platform identifying them with specific and unambiguous terminology and labels. In this case, the subdivision of the monument in hierarchical levels and classification of parts follows the formal and structural composition of the Neptune Fountain from a typological, morphological and constructive point of view.

The segmentation method has therefore divided the whole complex into levels depending on elevation (Level 0 to 5 and Underground Level), clearly identified by stylistic/compositional differences and morphological characteristics (Elements) and composition (Constitutive Material). In this way, any information can be easily connected with a determined geometry at different level of detail (granularity) and quickly found through web query by users (Apollonio, 2016).

In fact, the conceptual basis provides the connection between an operation by the responsible restoration operator and the $3 \mathrm{D}$ element, which links information and model for information storage and advanced search (Figure 12).

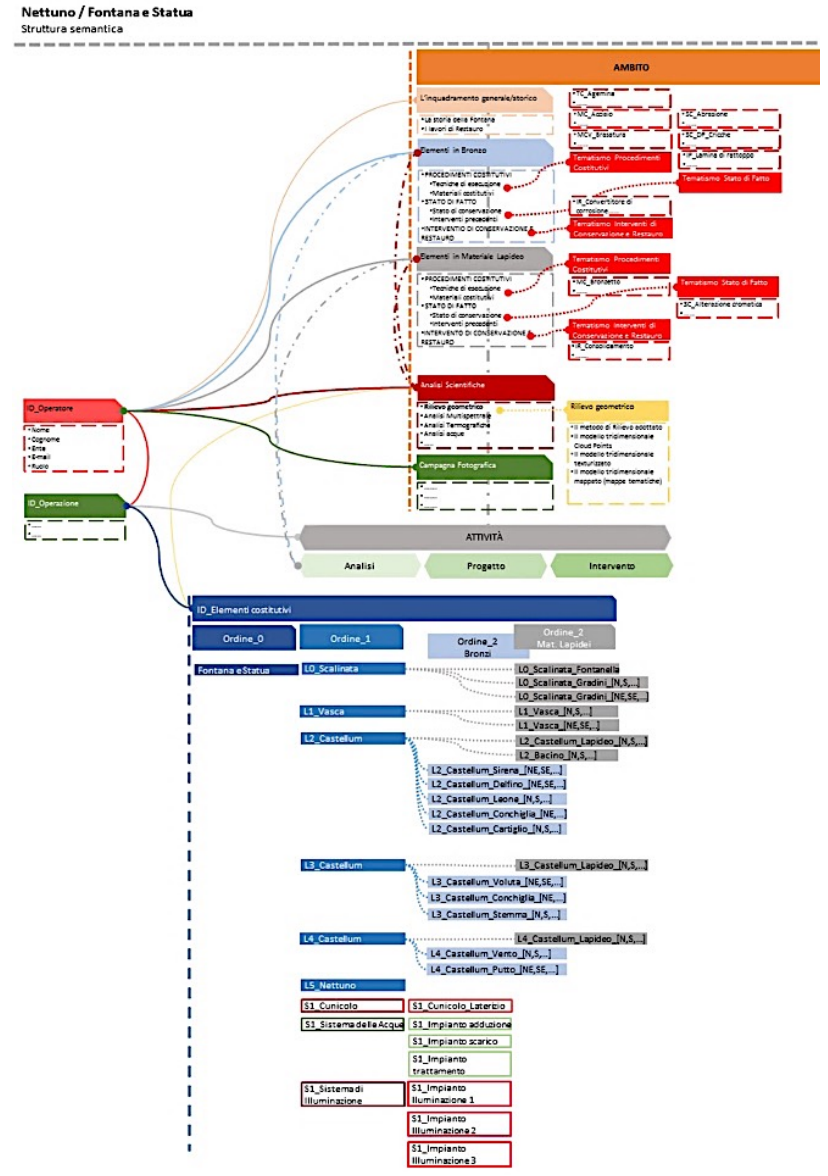

Figure 12. Data modelling: semantic partition of the 3D model and relationships between operators and processes during the restoration project of the fountain 


\section{CONCLUSIONS}

The paper describes the image-based 3D model construction of the Neptune fountain starting from high-resolution imagery taken exclusively for documentation purposes before the recent restoration activities of the monument.

The 3D digital model obtained is used for several applications supporting restoration activities.

Other applications are possible demonstrating the high flexibility and the efficiency of the automated photogrammetric process and of our customizations in the monuments conservation field.

\section{ACKNOWLEDGEMENTS}

This work was supported by the Bologna City Council. The authors are thankful to Fabio Andreon and Manuela Faustini (Municipality of Bologna); Gisella Capponi (Director of ISCR); Fabio Aramini, Dora Catalano, Patrizia Governale, Vilma Basilissi (ISCR), Pier Paolo Diotallevi, Gabriele Bitelli, Maurizio Mancini, Francesco Ceccarelli, Giovanni Naldi, Sandra Cristino, Elisa Franzoni, Cristiana Bragalli, Claudio Galli (University of Bologna); Marco Callieri, Matteo Delle Piane, Federico Ponchio, Roberto Scopigno (CNR-ISTI); LaMoViDA Lab (Dept. of Architecture, University of Bologna) for the 3D printing of the missing piece of the Neptune's trident; LUCE Laboratory (Department of Design, Politecnico di Milano) for the collaboration in the lighting analysis and design.

\section{REFERENCES}

Agisoft, 2017. www.agisoft.com.

Apollonio, F.I., 2016, Classification Schemes for Visualization of Uncertainty in Digital Hypothetical Reconstruction. Lecture Notes in Computer Science, Vol. 10025, pp. 173-197.

Apollonio, F.I., Ballabeni, A., Gaiani, M., Remondino, F., 2014. Evaluation of feature-based methods for automated network orientation. Int. Arch. Photogr. Remote Sens. Spatial Inf. Sci., Vol.XL(5), pp. 47-54.

Apollonio, F.I., Gaiani, M., Foschi, R., 2016. New water for the Neptune Fountain in Bologna: simulation of the design of the multi-jet system. Disegnare idee immagini, N. 53, pp. 68-79.

Apollonio, F.I., Gaiani, M., Basilissi, W., Rivaroli, L., 2017. Photogrammetry driven tools to support the restoration of open-air bronze surfaces of sculptures: an integrated solution starting from the experience of the Neptune Fountain in Bologna, Int. Arch. Photogramm. Remote Sens. Spatial Inf. Sci., Vol. XLII-2/W3, pp. 47-54.

Callieri M., Chica A., Dellepiane M., Besora I., Corsini M., Moyés J., Ranzuglia G., Scopigno R., Brunet P., 2011. Multiscale acquisition and presentation of very large artifacts: The case of Portalada. $A C M J O C C H$, Vol. 4(4).

CloudCompare, 2017. http://www.danielgm.net/cc/.

Comune di Bologna, 2017, http://nettuno.comune.bologna.it/.

DCRaw, 2017. http://www.cybercom.net/ dcoffin/dcraw/.

Gaiani, M., 2015. I portici di Bologna. BUP, Bologna.

Gaiani, M., Apollonio, F. I., Ballabeni, A., Remondino, F., 2016a. A technique to ensure color fidelity in automatic photogrammetry. Colour and Colorimetry. Multidisciplinary Contributions, Vol. XII B, pp. 53-66.

Gaiani, M., Remondino, F., Apollonio, F. I., Ballabeni, A., 2016b. An Advanced Pre-Processing Pipeline to Improve Automated Photogrammetric Reconstructions of Architectural Scenes. Remote Sensing, Vol. 8(3), 178.

Gonizzi Barsanti, S., Guidi, G., 2017. A geometric processing workflow for transforming reality-based 3D models in volumetric meshes suitable for FEA, Int. Arch. Photogramm. Remote Sens. Spatial Inf. Sci., Vol. XLII-2/W3, pp. 331-338.
Green, P., MacDonald, L.W., 2002. Colour Engineering: Achieving Device Independent Colour. John Wiley \& Sons, Hoboken, NJ.

Hong, G., Luo, M.R., Rhodes, P.A., 2001. A Study of Digital Camera Colorimetric Characterization Based on Polynomial Modeling. Color Research and Application, 26(1), pp. 76-84.

Hamed, A., Abdelhafiz, A., 2016. Simplified Approach to Convert 3D Laser Scanning Models to FEM-Models Applied on Ancient Statue in Egypt. Heritage 2016. $5^{\text {th }}$ international Conference on Heritage and Sustainable Development, pp. 1317-1327.

Lempitsky, V., Ivanov, D., 2007. Seamless Mosaicing of Image-Based Texture Maps. IEEE CVPR proceedings, pp. 1-6.

Lowe, D., 2004. Distinctive image features from scale-invariant keypoints. IJCV, Vol. 60(2), pp. 91-110.

Markiewicz, J.S., Podlasiak, P., Zawieska, D., 2015. Attempts to automate the process of generation of orthoimages of objects of Cultural Heritage, Int. Arch. Photogramm. Remote Sens. Spatial Inf. Sci., Vol. XL5/W4, pp. 393-401.

Mavromati, D., Petsa, E., Karras, G., 2002, Theoretical and practical aspects of archaeological orthoimaging, Int. Arch. Photogramm. Remote Sens. Spatial Inf. Sci., Vol. 34, pp. 413-418.

McCamy, C.S., Marcus, H., Davidson, J.G.,1976. A Color Rendition Chart. Journal of Applied Photographic Engineering, 11(3), pp. 95-99.

Meshlab, 2017. http://www.meshlab.net/.

Morel, J-M., Yu, G., 2009. ASIFT: a new framework for fully affine invariant comparison. SIAM Journal on Imaging Sciences, Vol. 2(2), pp. 438-469.

Muja, M., Lowe, D., 2009. Fast Approximate Nearest Neighbours with Automatic Algorithm Configuration. International Conference on Computer Vision Theory and Applications (VISAPP), pp. 331-340.

Pharr, M., Humphreys, G. 2016. Physically based rendering: from theory to implementation. Morgan Kaufmann, San Francisco.

Rossi, M., Marini, D., Rizzi, A., 2004. Methods and application for photorealistic rendering and lighting of ancient buildings, Journal of Cultural Heritage, Vol. 5(3), pp. 291-300.

Scopigno, R., Cignoni, P., Pietroni, N., Callieri, M., Dellepiane, M., 2017. Digital Fabrication Techniques for Cultural Heritage: A Survey. Computer Graphics Forum, Vol. 36, pp. 6-21.

Sederberg, T.-W., Zheng, J., Bakenov, A., Nasri, A., 2003. T-Splines and T-NURCCs. ACM Transactions on Graphics, Vol. 22(3), pp. 477-484.

Tucci, G., Guardini, N., 2014. Rilevo e modellazione 3D a supporto dell'analisi strutturale: un approccio metodologico e sostenibile per il patrimonio architettonico. Atti $18^{\circ}$ Conferenza ASITA, pp. 1197-209.

Tuttle, R.J., 2001. Piazza Maggiore. Studi su Bologna nel Cinquecento. Marsilio, Venezia.

Tuttle, R.J., 2015. The Neptune fountain in Bologna. Bronze, marble and water in the making of a papal city. Brepols-Harvey Miller, New York.

Wu, C., Agarwal, S., Curless, B., Seitz, S.M., 2011. Multicore Bundle Adjustment, CVPR Proceedings, IEEE Computer Society, pp. 30573064 . 